\title{
Mechanism of apoptosis induced by Mcl-1 inhibitor UMI-77 on gallbladder carcinoma GBC-SD cells
}

\author{
Shengbin Zhang, Baoqin Liu, Changcheng Dong, Bing Li* \\ Department of Hepatobiliary Surgery, Baogang Hospital, Baotou, Inner Mongolia, China
}

Received: January 6, 2019

Accepted: February 12, 2019 Online Published: March 10, 2019

DOI: $10.5430 /$ dcc.v6n1p1

URL: https://doi.org/10.5430/dcc.v6n1p1

\begin{abstract}
Objective: To investigate the mechanism of apoptosis induced by myeloid cell leukemia-1 (Mcl-1) inhibitor UMI-77 on gallbladder carcinoma GBC-SD cells.

Methods: GBC-SD cells were treated with different concentrations of UMI-77. GBC-SD cell proliferation and apoptosis were detected by MTT assay and Annexin V/PI. The expressions of Mcl-1, Bcl-2, Bcl-xL, Bax, Bak, cleaved-caspase 9, cleaved-caspase 3 and cleaved-PARP proteins in GBC-SD cells treated with UMI-77 were detected by Western blotting.

Results: The results of MTT showed that different concentrations of UMI-77 had different inhibitory effects on cell proliferation of GBC-SD cells in a dose-dependent and time-dependent manner. Annexin V/PI results showed that the apoptosis rate was increasing gradually with the increase of UMI-77 concentration in a dose-dependent manner. Western blotting results showed that the expression of anti-apoptotic protein Mcl-1 was significantly decreased $(p<.05)$, and the expressions of Bax and Bak proteins were significantly increased respectively $(p<.05)$, but there were no significant changes in the expressions of Bcl-2 and Bcl-xL proteins, and the expression levels of cleaved-caspase 9, cleaved-caspase 3 and cleaved-PARP proteins were significantly increased $(p<.05)$ in $24 \mathrm{~h}$ after GBC-SD cells were treated with $10 \mu \mathrm{mol} / \mathrm{L}$ of UMI-77.

Conclusions: Mcl-1 inhibitor UMI-77 can induce the apoptosis of GBC-SD cells in a dose-dependent manner through the caspase-mediated endogenous apoptosis pathway. Therefore, Mcl-1 may become a new therapeutic target in the research on gallbladder cancer.
\end{abstract}

Key Words: Mcl-1 inhibitor, UMI-77, Gallbladder carcinoma, Apoptosis

\section{INTRODUCTION}

Gallbladder carcinoma is one of the most common malignant tumors which occur in the biliary system, accounting for $2 / 3$ of the biliary tumors. Nevertheless, most of the patients are found to be in the middle or the terminal stage of cancer with local infiltration or tissue transfer when they are diagnosed as gallbladder carcinoma due to unclear pathogenesis, the lack of specific clinical manifestations and concealed onset. ${ }^{[1,2]}$ One of important symbols for the occurrence and develop- ment of tumors is the disorder of cell apoptosis, which is also one of factors that tumors are not sensitive to radiotherapy and chemotherapy. Therefore, to induce the apoptosis of tumor cells is a type of mechanism for the treatment of tumors. ${ }^{[3,4]}$ The apoptosis pathway can be subdivided into the exogenous pathway and the endogenous pathway. B-cell lymphoma 2 (Bcl-2) and myeloid cell leukemia-1 (Mcl-1) participate in the endogenous apoptosis pathway as anti-apoptotic proteins. ${ }^{[5]}$ In the aspect of cytology, the develChina.

*Correspondence: Bing Li; Email: 652287462@qq.com; Address: Department of Hepatobiliary Surgery, Baogang Hospital, Baotou, Inner Mongolia, 
opment of gallbladder carcinoma results from the disorder of the expression of cell proliferation- and apoptosis-associated factors. Gallbladder carcinoma cells show overexpression of anti-apoptotic factors Bcl-2, Mcl-1 and B-cell lymphoma extra large (Bcl-xL), with a low expression of pro-apoptotic factors Bcl-2 assaciated X protein (Bax) and Bcl-2 homologous antagonist killer (Bak). With the help of its BH3 domain, anti-apoptotic factors can bind to pro-apoptotic factors to form into heterodimers, protecting cancer cells from entering into apoptosis programme to inhibit the apoptosis of cancer cells and promote the development of gallbladder carcinoma. ${ }^{[6,7]}$ In recent years, researchers have synthesized various small-molecule inhibitors targeting anti-apoptotic proteins from the research of Bcl-2 and Mcl-1. The application of inhibitors alone or in combination with chemotherapy drugs has a good therapeutic effect. ${ }^{[8]}$ In 2014, with the application of structural biology and high throughput screening, Abulwerdi et al. ${ }^{[9]}$ identified a type of small-molecule inhibitor of Mcl-1, called UMI-77. It can selectively bind to Mcl-1 to rival the anti-apoptotic function of Mcl-1 and play a role in inducing apoptosis and inhibiting cell growth. This research applies UMI-77 to gallbladder carcinoma GBC-SD cells to explore the mechanism of UMI-77 on inducing the apoptosis of gallbladder carcinoma cells, with the aim of providing a theoretical basis for clinical application.

\section{MATERIALS AND METHODS}

\subsection{Materials}

Human gallbladder carcinoma cell line GBC-SD was provided by Cell Bank, Shanghai Institutes for Biological Sciences, Chinese Academy of Sciences. Mcl-1 inhibitor UMI77 was purchased from Selleck (USA); Annexin V/PI double staining kits were merchandised from Beijing Jingmei Biotechnology Co., Ltd.; RPMI-1640 medium and fetal calf serum (FBS) were purchased from Invitrogen (USA); cell proliferation assay kits were acquired from Sigma (USA). Rabbit anti-human polyclonal antibodies Mcl-1 (ab28147), Bcl-2 (ab59348), Bcl-xL (ab2568), Bax (ab53154), Bak (ab69404), cleaved cysteinyl aspartate specific proteinase (cleaved-caspase 9) (ab2324), cleaved-caspase 3 (ab2302), rabbit anti-human monoclonal antibodies cleaved poly ADPribose polymerase (cleaved-PARP) (ab32064), and goat antirabbit secondary antibody (ab6721) were purchased from Abcam (UK).

\subsection{Cell culture}

Conventionally, human gallbladder carcinoma GBC-SD cells were cultured in RPMI-1640 medium containing 10\% FBS (fetal bovine serum) and placed into the incubator $\left(37^{\circ} \mathrm{C}, 5 \%\right.$ $\mathrm{CO}_{2}$ ). Cells in the logarithmic phase were taken and used in the experiment.

\subsection{MTT assay of cell proliferation}

Cells in the logarithmic phase were inoculated in 96-well cell culture plates with a seeding density of $1 \times 10^{6}$ cells/well, the volume of medium in each well was $100 \mu \mathrm{l}$. UMI-77 at gradient concentrations of $0,5,10,15,20$ and $30 \mu \mathrm{mol} / \mathrm{L}$ were added into each well respectively. Each group was made in quintuple. Each group of cells were cultured for $12 \mathrm{~h}, 24 \mathrm{~h}$ and $48 \mathrm{~h}$, and then each well was added into $20 \mu \mathrm{l}$ of MTT solution. The supernatant was removed carefully after another 3-6 h cell culture, with $100 \mu \mathrm{l}$ of formazan solution added into each well for incubation. OD value of each well was measured by the microplate reader, with the average of OD values at the same time point taken from that of OD values from 5 parallel holes. Cell survival rate $(\%)=(\mathrm{OD}$ value of each concentration group/OD value of 0 $\mu \mathrm{mol} / \mathrm{L}$ group) $\times 100 \%$.

\subsection{The detection of cell apoptosis by flow cytometry (FCM)}

Cells in the logarithmic phase were inoculated in 24-well cell culture plates with a seeding density of $1 \times 10^{5}$ cells/well. Up to the next day, if the proportion of cell fusion exceeded $80 \%$, the cells were treated with $0,5,10,15,20$ and $30 \mu \mathrm{mol} / \mathrm{L}$ of UMI-77 respectively. Each group was made in triplicate. Cells in each group were cultured respectively for $24 \mathrm{~h}$, collected and added with PBS for resuspension and centrifugation. Besides, these cells were resuspended in $300 \mu 11 \times$ binding buffer, added by $5 \mu \mathrm{l}$ of Annexin V-FITC and labeled by $5 \mu \mathrm{l}$ of propodium iodide (PI). The mixture was fully mixed and then incubated in a light-proof place for 20 min. The FCM was used to detect cell apoptosis.

\subsection{The detection of the expression of proteins by West- ern blotting}

Western blotting can be used to detect the expressions of Mcl-1, Bcl-2, Bcl-xL, Bax, Bak, cleaved-caspase 9, cleavedcaspase 3 and cleaved-PARP in each group of GBC-SD cells at the optimal concentration and the optimal time point. Each group of cells were collected, washed with PBS and added by lysis buffer. After cell lysis, the supernatant was collected by means of centrifugation. Bicinchoninic acid (BCA) was used to measure the concentration of proteins. Each well was added into $20 \mu \mathrm{l}$ of sample proteins. After $12 \%$ sodium dodecyl sulfate polyacrylamide gel electrophoresis (SDS-PAGE), proteins were transferred to PVDF, which was blocked for $1 \mathrm{~h}$ at room temperature in 5\% skim milk, added by primary antibodies (diluted under the ratio of 1:1,000) and incubated overnight at $4{ }^{\circ} \mathrm{C}$. After washing with TBST for 3 times, human specific secondary antibodies (diluted under the ratio of 1:5,000) were added, with the incubation continued for $1 \mathrm{~h}$ at room temperature. PVDF was fully rinsed out and 
developed for photomicrograph. Each group was made in triplicate, and Image $\mathbf{J}$ software was used to make a quantitative gray-degree analysis of the bands, with the average value taken.

\subsection{Statistical treatment}

The experimental data were represented by mean \pm standard deviation, $t$-test was applied to the comparison between two groups and one-way ANOVA was applied to the comparison among groups. SPSS 19.0 statistical software was used in the statistical treatment, and $p<.05$ indicated the difference was of statistical significance.

\section{Results}

\subsection{The inhibition of UMI-77 on GBC-SD cell prolifera- tion}

According to Figure 1, MTT results showed that different concentrations of UMI-77 acted on GBC-SD cells for different time durations, the survival rate of cells in each group was significantly decreased in a dose-dependent and timedependent manner. Compared to cells with no UMI-77 addition of the corresponding period, cells treated with no less than $10 \mu \mathrm{mol} / \mathrm{L}$ of UMI-77 for $12 \mathrm{~h}, 24 \mathrm{~h}$ and $48 \mathrm{~h}$, showed a significant decrease in the survival rate $(p<.05)$, i.e., UMI77 can significantly inhibit cell proliferation. UMI-77 at the concentration of $10 \mu \mathrm{mol} / \mathrm{L}$ was applied to the follow-up study, and the treatment time was $24 \mathrm{~h}$.

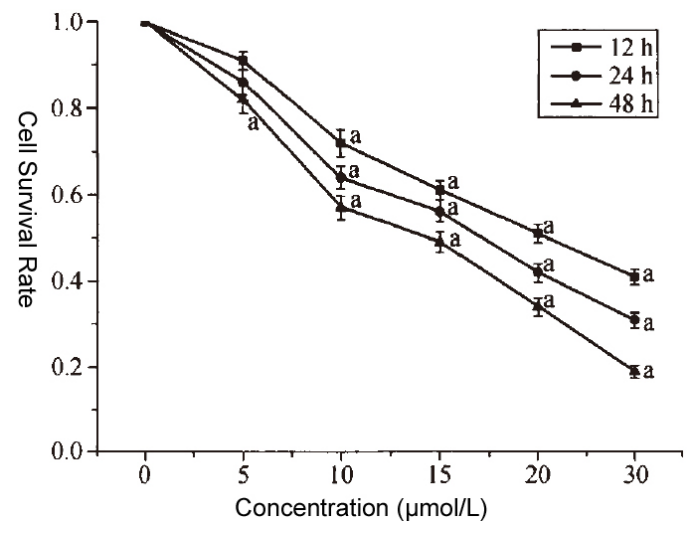

Figure 1. Inhibitory effects of UMI-77 at different concentrations on the proliferation of GBC-SD cells (Compared with $0 \mu \mathrm{mol} / \mathrm{L}$ group, a $p<.05$ )

\subsection{The effect of UMI-77 on promoting GBC-SD cell apoptosis}

After GBC-SD cells were treated with UMI-77 at the concentration of $0,5,10,15,20$ and $30 \mu \mathrm{mol} / \mathrm{L}$ respectively for $24 \mathrm{~h}, \mathrm{FCM}$ was applied to the detection of cell apoptosis. The results (see Figure 2) showed that, the apoptosis rate was gradually increased with the increase of drug concentration in a dose-dependent manner. Compared to cells with no UMI-77 addition of the corresponding period, cells treated with UMI-77 at different concentrations for $24 \mathrm{~h}$, showed a significant increase in the apoptosis rate $(p<.05)$, i.e., UMI77 at different concentrations can significantly promote cell apoptosis (see Figure 3).
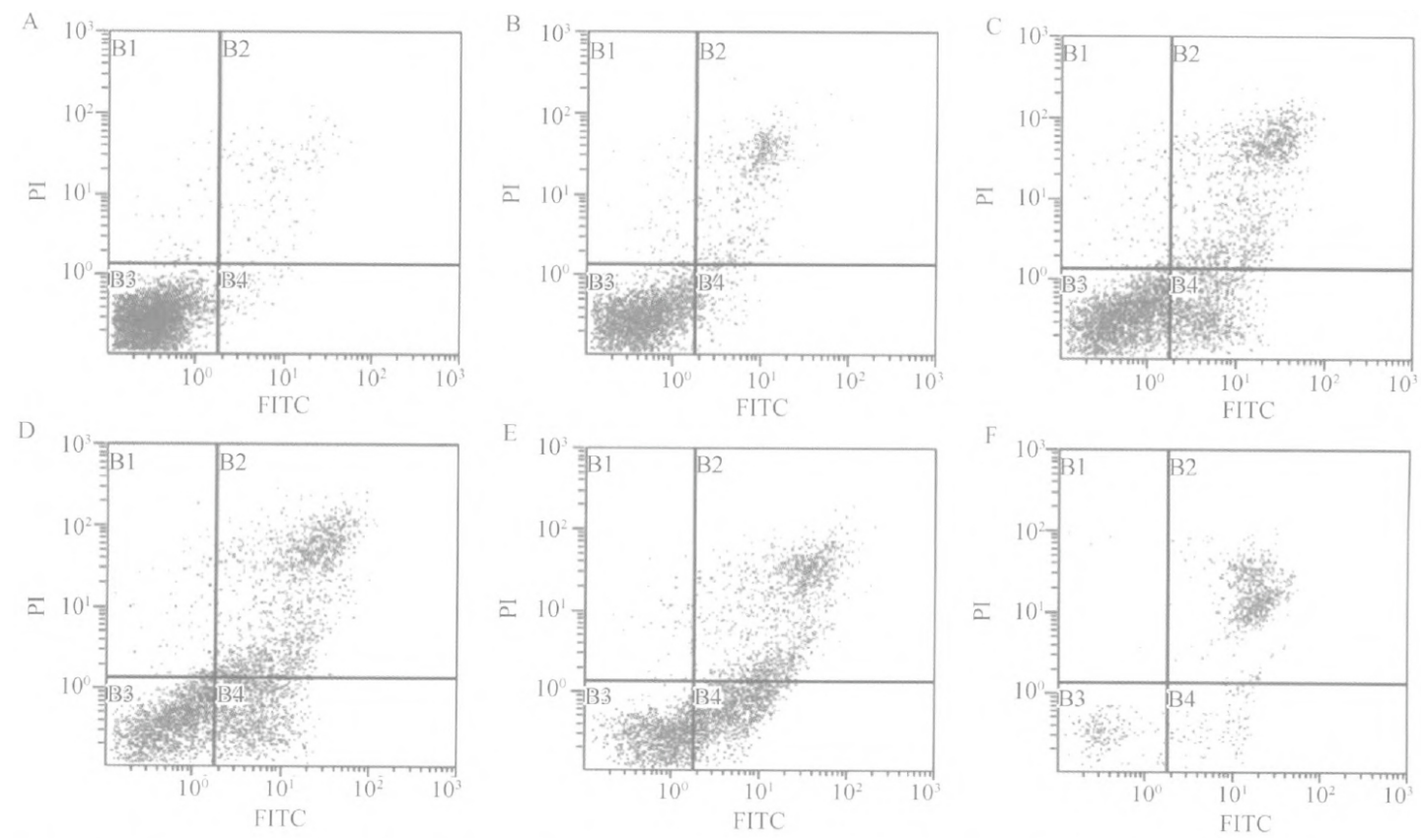

Figure 2. Apoptosis rates of GBC-SD cells treated with UMI-77 at different concentrations for $24 \mathrm{~h}$

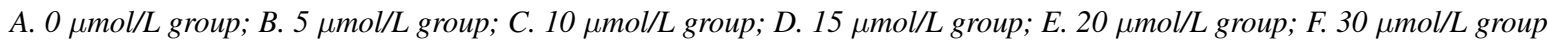




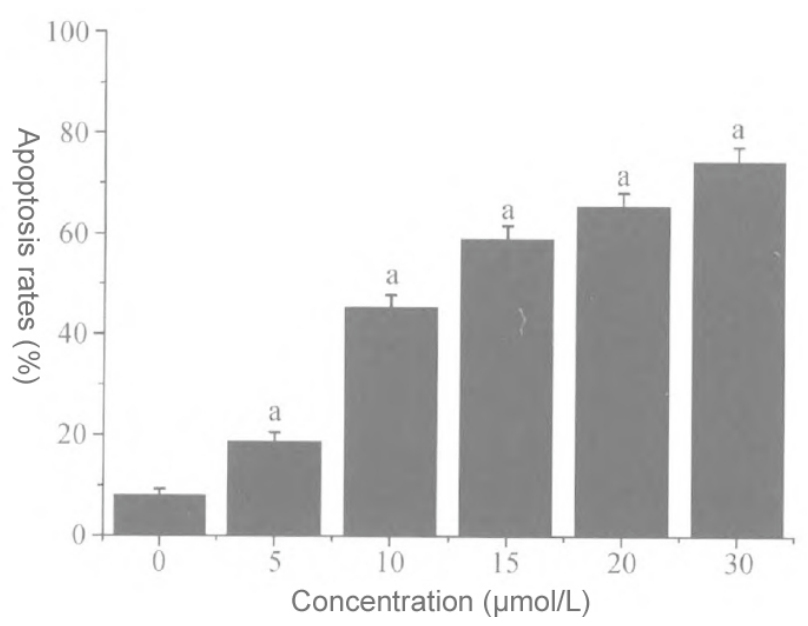

Figure 3. Apoptosis rates of GBC-SD cells treated with UMI-77 at different concentrations for $24 \mathrm{~h}$

Compared with 0 rmol/L group, a $p<.05$

3.3 The effect of UMI-77 on the expressions of Mcl-1, Bcl-2, Bcl-xL, Bax and Bak in GBC-SD cells

Western blotting was used to detect the expressions of antiapoptotic proteins Bcl-2, Bcl-xL and Mcl-1, pro-apoptotic proteins Bax and Bak before and after GBC-SD cells were treated with UMI-77 at the concentration of $10 \mu \mathrm{mol} / \mathrm{L}$ (see Figure 4). Compared with the untreated group, after GBCSD cells were treated with UMI-77 at the concentration of $10 \mu \mathrm{mol} / \mathrm{L}$ for $24 \mathrm{~h}$, the expression of Mcl-1 was significantly decreased $(p<.05)$, and the expressions of Bax and Bak were significantly increased $(p<.05)$, but the expressions of Bcl-2 and Bcl-xL showed no obvious changes.

\subsection{The effect of UMI-77 on the expressions of cleaved- caspase 9, cleaved-caspase 3 and cleaved-PARP in GBC-SD cells}

Western blotting was applied to the detection of the expressions of cleaved-caspase 9, cleaved-caspase 3 and cleavedPARP proteins before and after GBC-SD cells were treated with UMI-77 at the concentration of $10 \mu \mathrm{mol} / \mathrm{L}$ (see Figure 5). Compared with the untreated group, after GBC-SD cells were treated with UMI-77 at the concentration of $10 \mu \mathrm{mol} / \mathrm{L}$ for $24 \mathrm{~h}$, the expressions of active fragments cleaved-caspase 9, cleaved-caspase 3 and cleaved-PARP were significantly increased, and the difference was of statistical significance $(p<.05)$.
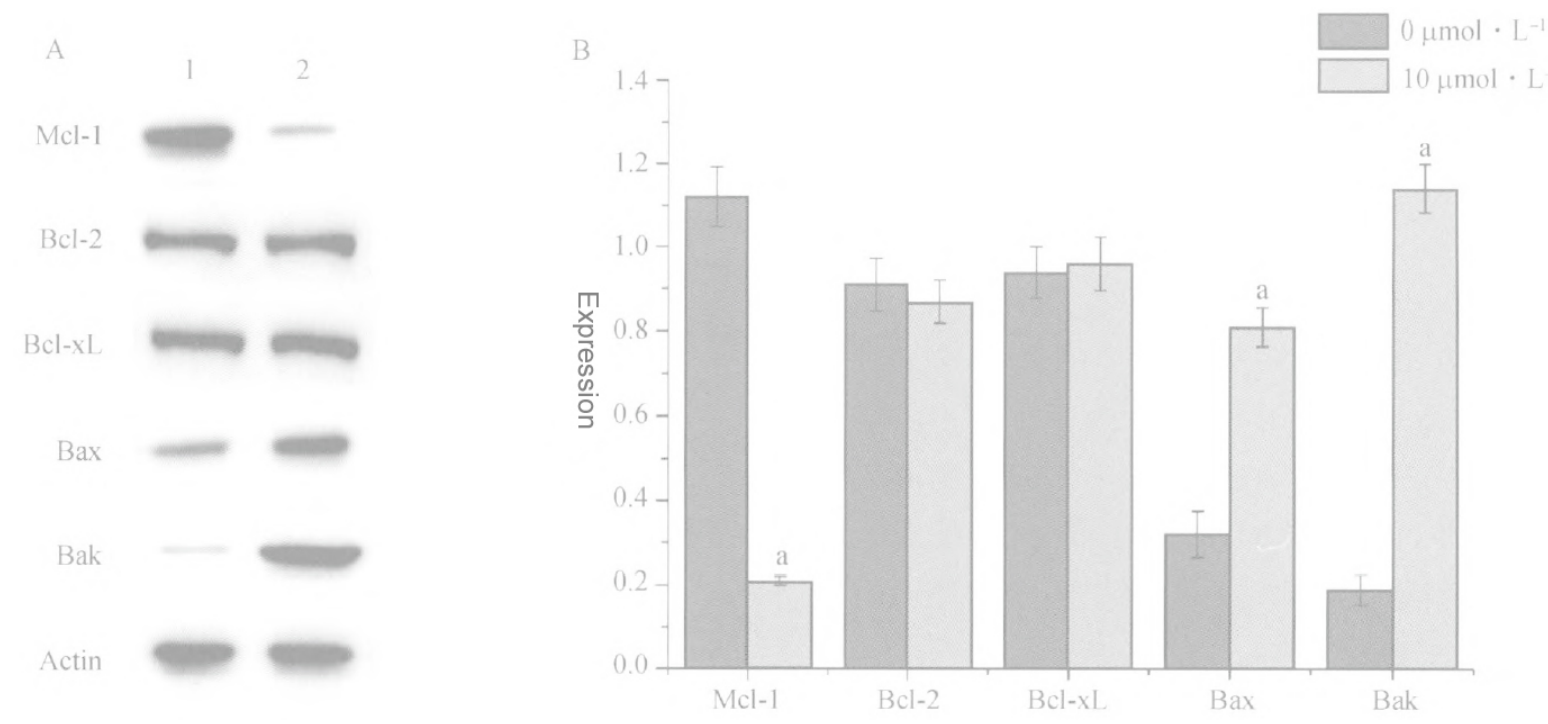

Figure 4. The effect of UMI-77 on the expressions of Mcl-1, Bcl-2, Bcl-xL, Bax and Bak proteins in GBC-SD cells 1 stands for $0 \mu \mathrm{mol} / \mathrm{L}$ group; 2 represents $10 \mu \mathrm{mol} / \mathrm{L}$ group; In comparison with $0 \mu \mathrm{mol} / \mathrm{L}$ group, a $p<.05 ;$ A. The detection of Mcl-1, Bcl-2, Bcl-xL, Bax and Bak proteins by Western blotting; B. The expressions of Mcl-1, Bcl-2, Bcl-xL, Bax and Bak proteins

\section{Discussion}

As a type of the most common malignant tumor in the biliary system, gallbladder carcinoma accounts for $2 / 3$ of the biliary tumors. Nevertheless, most of the patients are found to be in the middle or the terminal stage of cancer with local infiltration and tissue transfer when they are diagnosed as gallbladder carcinoma due to unclear pathogenesis, the lack of specific clinical index and conceal onset. ${ }^{[1]}$ Meanwhile, gallbladder carcinoma is prone to drug resistance, and is not sensitive to radiotherapy and chemotherapy. Tumor prognosis is not promising. ${ }^{[10]}$ Therefore, it is crucial to make a research to explore the therapeutic target associated with gallbladder carcinoma. Mcl-1 is a type of anti-apoptotic factors in Bcl-2 family, with a powerful and unique function and low 
half-life period. It is highly expressed in various tumor tissues and cells. ${ }^{[11,12]}$ When Mcl-1 is over-expressed in tumor tissues, it can significantly suppress the anti-tumor effect of inhibitors towards Bcl-2. ${ }^{[13]}$ Starting with Mcl-1, researchers have synthesized various small-molecule inhibitors target- ing anti-apoptotic proteins. The application of inhibitors alone or in combination with chemotherapy drugs has a good therapeutic effect. ${ }^{[8]}$ These researches indicate that Mcl-1 is probably a new target in the treatment of tumors.

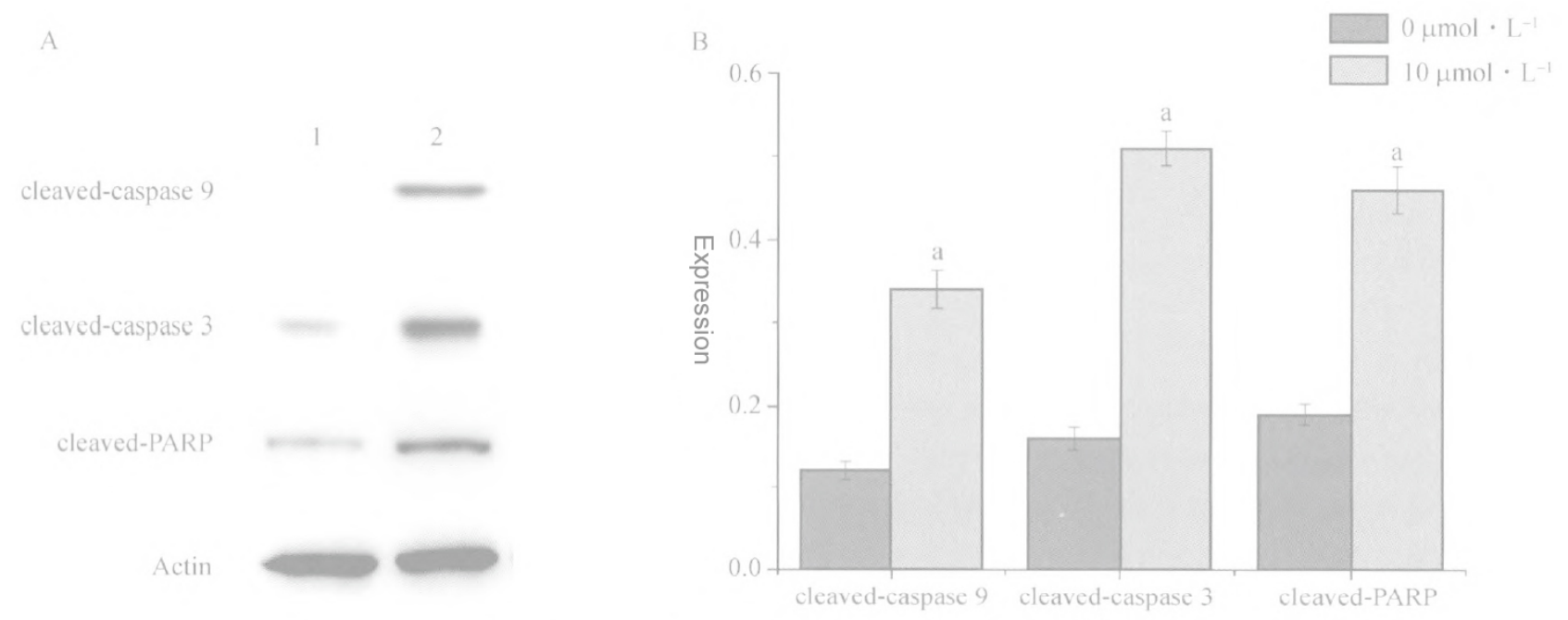

Figure 5. The effect of UMI-77 on the expressions of cleaved-caspase 9, cleaved-caspase 3 and cleaved-PARP proteins in GBC-SD cells

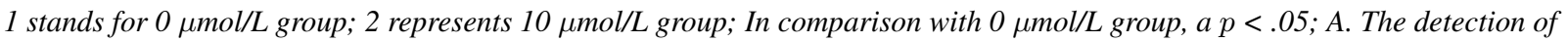
cleaved-caspase 9, cleaved-caspase 3 and cleaved-PARP proteins by Western blotting; B. The expressions of cleaved-caspase 9 , cleaved-caspase 3 and cleaved-PARP proteins

One of important symbols for the occurrence and development of tumors is the disorder of cell apoptosis, which is also one of factors that tumors are not sensitive to radiotherapy and chemotherapy. Therefore, to induce the apoptosis of tumor cells is a type of mechanism for the treatment of tumors. ${ }^{[3,4]}$ In recent years, there have apppeared an increasing number of researches on inhibitors of anti-apoptotic proteins. Most of the researches focus on ABT-737 and its oral derivative ABT-263, the small-molecule inhibitors of Bcl-2 protein. This type of inhibitors has an inhibitory effect on the activity of most anti-apoptotic proteins, but poorly binds to Mcl-1, so that tumor cells with a high expression of $\mathrm{Mcl}-1$ are prone to drug resistance to ABT-737. Therefore, it is of critical significance for the treatment of tumors to make a research on the inhibitor of Mcl-1 protein. ${ }^{[14,15]}$ UMI-77 is a smallmolecule inhibitor targeting Mcl-1. It can selectively bind to Mcl-1 to rival the anti-apoptotic function of Mcl-1 and play a role in promoting apoptosis. ${ }^{[9]}$ Studies by Xueping Zhu et al. ${ }^{[16]}$ showed that the mechanism of inducing the apoptosis of gastric carcinoma cell MGC-803 by UMI-77 is probably to activate endogenous apoptosis pathway. In this research, UMI-77 with different concentrations was applied to the treatment of gallbladder carcinoma GBC-SD cells. The results showed that UMI-77 can significantly inhibit the Published by Sciedu Press proliferation of GBC-SD cells and promote the apoptosis of GBC-SD cells in a dose-dependent and time-dependent manner. After gallbladder carcinoma GBC-SD cells were treated with UMI-77 for $24 \mathrm{~h}$, the expressions of anti-apoptotic proteins Bcl-2 and Bcl-xL in Bcl-2 family showed no significant changes. Nevertheless, the expression of Mcl-1 was significantly reduced and the expressions of pro-apoptotic proteins Bax and Bak were obviously up-regulated. All these results futher identified that UMI-77 can selectively bind to Mcl-1, reduce the expression of $\mathrm{Mcl}-1$ in the cells and prevent the heterodimerization of $\mathrm{Mcl}-1 / \mathrm{Bax}$ and $\mathrm{Mcl}-1 / \mathrm{Bak}$ to increase the exoressions of Bax and Bak proteins and promote the apoptosis of tumor cells. In this research, after GBC-SD cells were treated with UMI-77 at the concentration of 10 $\mu \mathrm{mol} / \mathrm{L}$ for $24 \mathrm{~h}$, the expressions of active cleaved-caspase 9 , cleaved-caspase 3 and cleaved-PARP were significantly higher than those in the untreated cells. The difference ( $p$ $<.05)$ was of statistical significance. Endogenous apoptosis pathway, also called mitochondrial pathway, starts at mitochondria. It can recruit and activate caspase 9 by releasing apoptosis-associated factor, and then activate caspase 2 , caspase 3, caspase 6 and other factors to initiate cascade reactions of caspases, eventualy lead to cell apoptosis. ${ }^{[17]}$ When the apoptosis of tumor cells is inhibited, caspase 3, 
caspase 9 and PARP stays in an unactivated condition. After cells are treated with UMI-77, a series of stress responses will appear so that caspase (e.g., cleaved-caspase 3, cleavedcaspase 9) and PARP can be cleaved and activated by means of cascade reactions of caspases. Eventually, cell apoptosis will be induced through endogenous apoptosis pathway.

\section{Conclusions}

In summary, this research indicates that the small-molecule inhibitor UMI-77 targeting Mcl-1 can inhibit the proliferation of gallbladder carcinoma GBC-SD cells in a dose-dependent and time-dependent manner. Meanwhile, it can also induce the apoptosis of GBC-SD cells through caspase-mediated endogenous apoptosis pathway in a dose-dependent manner, the mechanism is probably realized by reducing the expression of Mcl-1. It is indicated that Mcl-1 can be considered as a new therapeutic target in the research on gallbladder carcinoma. Therefore, the specific mechanism on how the small-molecule inhibitor UMI-77 targeting Mcl-1 has a good anti-tumor effect needs to be further studied, which provides a new choice for anti-tumor treatment.

\section{FINANCIAL SUPPORT}

The research was funded by Natural Science Foundation of Inner Mongolia Autonomous Region (2013MS1102).

\section{CONFLiCTS OF INTEREST DisClosure}

The authors declare they have no conflicts of interest.

\section{REFERENCES}

[1] Hundal R, Shaffer EA. Gallbladder cancer: epidemiology and outcome. Clin Epidemiol. 2014; 6(1): 99-109. PMid:24634588.

[2] Bin Z, Jian T, Cibao Z, et al. Effect of HMGB1 on proliferation and apoptosis of gallbladder carcinoma cells and its mechanism. Modern Medical Journal. 2017; 45(10): 1422-1426.

[3] Hanahan D, Weinberg RA. Hallmarks of cancer: the next generation. Cell. 2011; 144(5): 646-674. PMid:21376230. https: //doi.org/10.1016/j.cell.2011.02.013

[4] Kelly G, Strasser A. The essential role of evasion from cell death in cancer. Adv Cancer Res. 2011; 111: 39-96. PMid:21704830. https://doi.org/10.1016/B978-0-12-385524-4.00002-7

[5] Anilkumar U, Prehn JHM. Anti-apoptotic BCL-2 family proteins in acute neural injury. Front Cell Neurosci. 2014; 8: 281 PMid:25324720. https://doi.org/10.3389/fncel.2014.002 81

[6] Jun N, Lipin C. Research progress on the mechanism of gallbladder carcinoma cell proliferation and apoptosis factors. Chinese Journal of Medicine. 2003; 38(10): 34-36.

[7] Jiahui F, Wei Y. A review of Bcl-2 family members and cardiomyocytes apoptosis. Modern Medical Journal. 2017; 45(7): 996-1000.

[8] Kiprianova I, Remy J, Milosch N, et al. Sorafenib Sensitizes Glioma Cells to the BH3 Mimetic ABT-737 by Targeting MCL1 in a STAT3-Dependent Manner. Neoplasia. 2015; 17(7): 564-573. PMid:26297434. https://doi.org/10.1016/j.neo.2015.07. 003

[9] Abulwerdi F, Liao C, Liu M, et al. A novel small-molecule inhibitor of mcl-1 blocks pancreatic cancer growth in vitro and in vivo. Mol Cancer Ther. 2014; 13(3): 565-575. PMid:24019208. https://doi.org/10.1158/1535-7163.MCT-12-0767

[10] Jain K, Sreenivas V, Velpandian T, et al. Risk factors for gallbladder cancer: a case-control study. Int J Cancer. 2013; 132(7): 1660-1666. PMid:22890893. https://doi .org/10.1002/ijc. 27777

[11] Perciavalle RM, Opferman JT. Delving deeper: MCL-1's contributions to normal and cancer biology. Trends Cell Biol. 2013; 23(1): 22-29. PMid:23026029. https://doi.org/10.1016/j.tcb. 20 12.08 .011

[12] Xia Y, Rui W, Kai X, et al. Knockdown of myeloid cell leukemia 1 gene inhibits the proliferation and promotes apoptosis of prostate cancer cells. Chinese Journal of Cellular and Molecular Immunology. 2017; 33(1): 62-66.

[13] Moldoveanu T, Follis AV, Kriwacki RW, et al. Many players in BCL-2 family affairs. Trends Biochem Sci. 2014; 39 (3): 101-110. PMid:24503222. https://doi.org/10.1016/j.tibs.2013.12 .006

[14] Jiahui Y, Yiren W, Nan Z, et al. Research progress of small-molecule Mcl-1 inhibitors on cancer therapy. Northwest Pharmaceutical Journal. 2016; 31(5): 547-552.

[15] Shenchun X, Junsong H, Yue L. Advances in small molecule kinase inhibitors for cancer treatment. Chinese Bulletin of Life Sciences. 2016; 7: 786-792.

[16] Xueping Z, Ping W, Linjie Z. Mechanism of apoptosis induced by Mcl-1 inhibitor UMI-77 on gastric cancer MGC-803 cells. Acta Universitatis Medicinalis Anhui. 2016; 51(4): 506-511.

[17] Xiaoyan L, Suzhen B. Research progress on caspase family. Inner Mongol Journal of Traditional Chinese Medicine. 2012; 31(2): 107. 